\title{
Performance of Chirped-FSK and Chirped-PSK in the Presence of Partial-band Interference
}

\author{
Ramen Dutta, Andre B. J. Kokkeler, Ronan v. d. Zee, Mark J. Bentum \\ University of Twente, Enschede, The Netherlands \\ email: R.dutta@utwente.nl, A.B.J.Kokkeler@utwente.nl, R.A.R.vanderZee@utwente.nl, \\ M.J.Bentum@utwente.nl,
}

\begin{abstract}
To improve interference robustness of wireless communication, spread spectrum techniques are often used. We use the chirp spreading technique along with FSK and PSK binary modulation schemes to obtain interference robust radio communication. The performance of chirped-FSK and chirpedPSK modulation through a white gaussian noise channel is simulated assuming a synchronized clock between transmitter and the receiver. We analyzed and simulated the error probability (BER) of the overall system in the presence of partial band of interference in the channel. The simulated BER is close to the estimated BER and they prove the superior performance of chirp-based modulation in the presence of interference.
\end{abstract}

Index Terms - Interference-robust, Chirped spread spectrum, FSK, PSK.

\section{INTRODUCTION}

$\mathrm{T}$ here are a large number of applications targeting for robust and energy-efficient Wireless Sensor Network (WSN) communication. There are large number of literatures improving the communication energy efficiency of the WSN to increase the battery lifetime such as [1]. However, interference robustness is not addressed enough although communication in most WSNs has to happen in the largely crowded ISM frequency bands [2]. A global ISM band in the frequency range from $2.4 \mathrm{GHz}$ to $2.5 \mathrm{GHz}$ is widely chosen for WSNs as a good tradeoff between antenna size and power consumption. This band of frequency is highly occupied by applications such as WLAN, zigbee, Bluetooth, cordless phone, wireless USB, microwave oven etc. The sensor network, therefore has to co-exists with one or more of these short range radio standards. A serious effect of the interference can occur if proper care is not taken into account [3]. Hence interference robustness from those signals of various standard is necessary for reliable communications between sensor nodes.

Among the ISM band standards, Wi-Fi (Wireless LAN) transmits signals with a largest bandwidth of $22 \mathrm{MHz}$. Where a cordless phone signal has a bandwidth of $10 \mathrm{MHz}$, all other signals are narrow band. So, a narrowband rejection scheme will still be effective against interference for most of the standard applications.

Basic binary modulation techniques are generally used in wireless sensor radio transceivers because of a simple and comparatively low power transceiver architecture. However, those techniques are prone to in-band interferences. Among

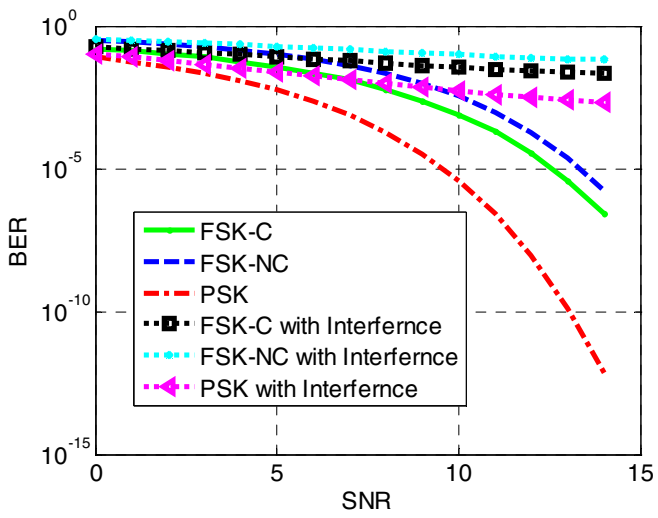

Figure 1 : Interference effect on BER of PSK and FSK (Coherent and non-coherent)

the binary modulation techniques, FSK and PSK schemes are most interference robust [4]. We simulated the BER performance of binary FSK (coherent and non-coherent) and PSK receiver for an AWGN channel including a partial band of interference. The BER curve of these modulation schemes gets deviated (Interference power $4 \mathrm{~dB}$ less than signal power, $10 \mathrm{MHz}$ wide and evenly spaced around the carrier frequency) as shown in Figure 1.

Though interference robustness is a common challenge in all wireless communication systems, it is even a bigger problem in wireless sensor network application, where available energy is very limited. Interference robust schemes which add significant amount of power, can be used in other highperformance communication systems but not in sensor networks because of limited energy resources. Therefore we need a simplified robustness scheme which can tradeoff other performance metrics such as bandwidth efficiency or data rate, but does not increase power consumption considerably.

\section{SPREAd SPECTRUM Using CHIRPED Clock}

Spread spectrum techniques are useful to mitigate narrow band interference $[5,6]$. The most popular spread spectrum techniques, direct sequence spread spectrum (DSSS) and frequency hopping spread spectrum (FHSS) can achieve high performance but at the cost of large power consumption [7]. Another technique, which is called the chirped spread spectrum, is proposed for RADAR [8] and communication [9] using surface acoustic wave (SAW) devices for spreading and dispreading the signal in the transmitter and receiver respectively. The external SAW device is not very suitable for wireless sensor nodes which intend to be as small as possible 


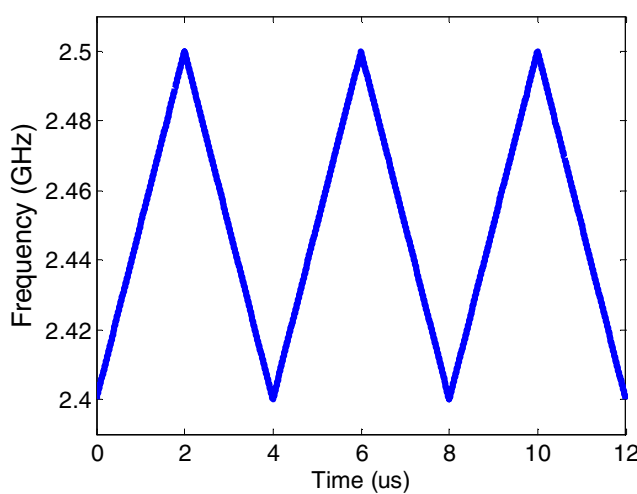

Figure 2 : Up-down chirp signal

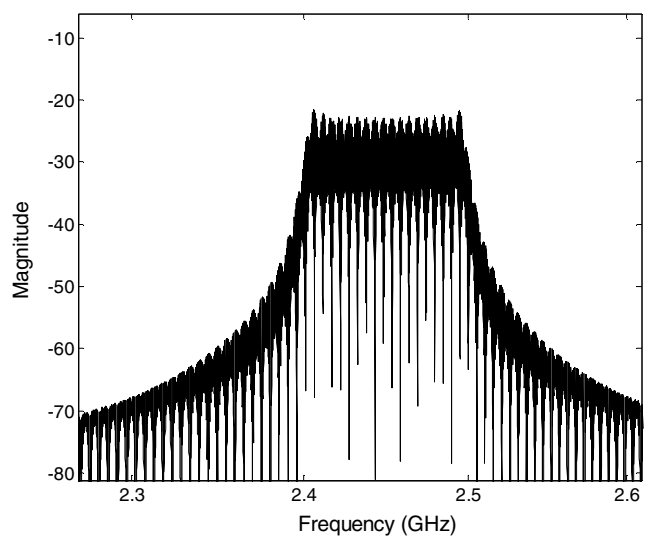

Figure 3 : Spectral spreading of chirp signals

in size. Moreover SAW devices can give a loss of 20 to $30 \mathrm{~dB}$ [10]. Instead of using SAW devices we generate a chirp clock by feeding a ramp voltage to a voltage controlled oscillator (VCO). This way, conventional radio mixers (multipliers) can be used to down-convert the chirped-carrier-modulated data.

\section{1) Chirped theory}

A clock is chirped when the frequency of the signal is changing with time. For a linear chirp, the rate of change is constant. The angular frequency can be represented as:

$$
\omega=\omega_{O} \pm \mu \cdot t
$$

where, '+' and '-' are used for up-chirp and down-chirp respectively. Non-linear chirps are those where $\mu$ is a function of time. For a continuous up-down chirp, shown in the Fig. 2, the starting frequency $\left(\omega_{o}\right)$ will be different for a up-chirp and a down-chirp.

$$
\omega(t)=\omega_{O}+\mu(t-n T) \quad \text { For even } \mathrm{n}=0,2,4 . .
$$

and,

$$
\omega(t)=\omega_{O}+B-\mu(t-n T) \quad \text { For odd } \mathrm{n}=1,3,5 . .
$$

For only one up-chirp signal, the instantaneous phase can be written from (1) as:

$$
\phi=\int \omega(t) d t=\omega_{O} t+\frac{1}{2} \mu t^{2}+c_{1}
$$

Therefore an up-chirp signal can be represented as:

$$
s_{U C}(t)=A \cos \left(\omega_{0} t+\frac{1}{2} \mu t^{2}+\phi_{1}\right)
$$

where ${ }_{l}$ is the initial phase of the signal.

\section{2) Robustness by spectrum spreading}

The interference rejection is achieved due to spectral spreading of the signal over the spreading bandwidth $\left(B_{C}\right)$. The ratio of these two bandwidths gives a measure of the interference robustness that can be achieved. If the interference bandwidth is $B_{i}$, the ratio equals:

$$
r_{W}=\frac{B_{i}}{B_{C}}
$$

Another performance parameter of spread spectrum communication is the time-bandwidth product, which is also called processing gain [7] which is defined as:

$$
G=B_{C} T
$$

where $T$ is the chirp time. The spectrum of a chirp pulse can be expressed accurately by Fresnel sine and cosine integrals [8]. A simplified and reasonable approximation for a chirp spectrum can be found in [11] for a time-bandwidth (T-B) product greater than 10 . The time-bandwidth product determines the spectral response of a chirp waveform. For example for a time-bandwidth product of $100,98 \%$ of the signal power remains within the chirp bandwidth. We used TB product of 200 and corresponding spectral response of an up-down chirp as shown in Figure 3.

\section{MODULATION}

\section{A. Chirp and BFSK}

We use a chirped clock as a carrier frequency and modulate the message in FSK style over the chirped frequency. The ' 1 'and ' 0 ' bit of the message can be represented by two chirps with the same frequency-time slope and separated by $\Delta f$ as shown in Fig. 4(a).

$$
\begin{aligned}
& s_{1}(t)=A \cos \left(2 \pi f_{1} t+\frac{1}{2} \mu t^{2}\right) \\
& s_{2}(t)=A \cos \left(2 \pi f_{2} t+\frac{1}{2} \mu t^{2}\right)
\end{aligned}
$$

The modulated signal will be switched between those two chirps depending on the message. An ideal modulator block diagram is shown in Fig. 4(b). Here the chirped clock is indicated by the input of the voltage controlled oscillator as it can be practically generated.

\section{1) Chirped-FSK demodulation}

An FSK receiver can be coherent or non-coherent. In the coherent receiver we assumed that the exact chirp functions (8) and (9) are available at the receiver after proper frequency and phase synchronization. This detection is based on correlation and integration over symbol time $\left(T_{b}\right)$ as shown in Figure 5.

In this case the optimum coherent FSK receiver used for chirped-FSK communication is realized. The probability of error (or bit error rate) of detection done by this coherent FSK receiver in the presence of white Gaussian noise can be expressed as [12]: 


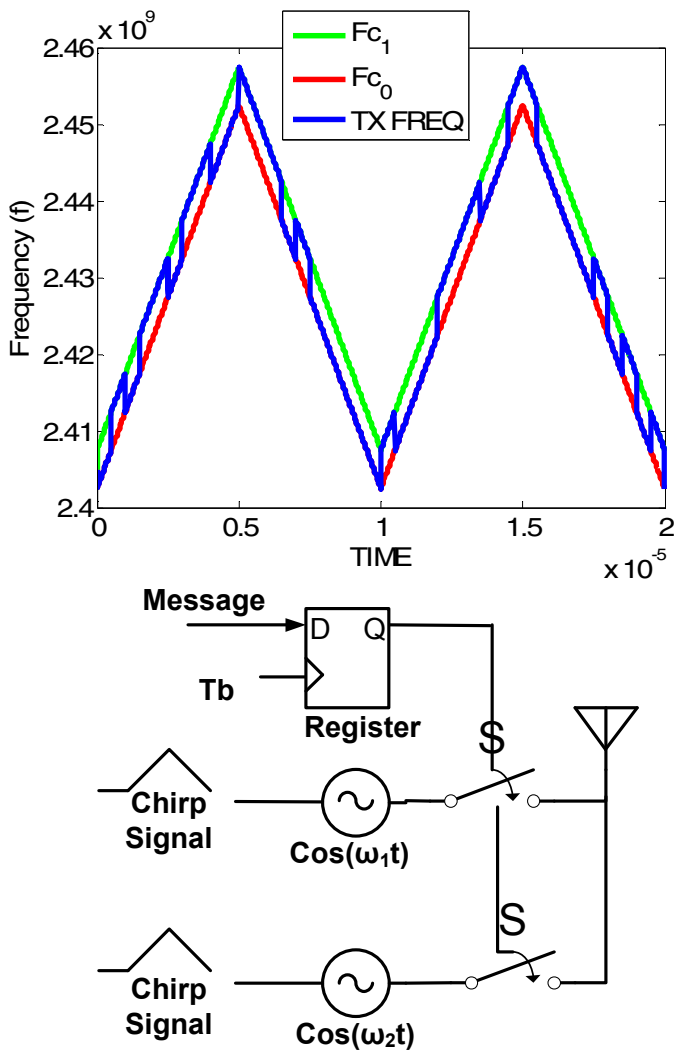

Figure 4 : chirped BFSK (a) signal and (b) ideal modulator

$$
P_{C F S K}=Q\left(\sqrt{\frac{E_{b}}{N_{O}}}\right)=Q(\sqrt{S N R})
$$

where $E_{b}$ is the energy per bit and $N_{o}$ is the power spectral density of white gaussian noise and Q(.) is Malcolm's Q function which is defined as (probability of a zero mean, one standard deviation gaussian distributed random function being between $x$ to infinity):

$$
Q(x)=\frac{1}{2 \pi} \int_{x}^{\infty} \exp \left(-\frac{u^{2}}{2}\right) d u
$$

The ratio of $E_{b} / N_{O}$ is the same as the signal to noise ratio $(S N R)$ assuming the signal and noise bandwidths are same. Now we assume partial-band Gaussian random interference with zero mean and with flat power spectral density (noiselike) is added in the channel. This interference is assumed to be present in a fraction of the total bandwidth achieved by the chirp-clock and absent (zero power) everywhere else.

A noise-like Gaussian distributed interference over the carriers of an FSK signal can increase the noise level and increase the error probability. Assuming an interference power spectral density of $I_{O}$ we can modify the probability of error for FSK in the presence of interference as:

$$
P_{C F S K_{-} I}=Q\left(\sqrt{\frac{E_{b}}{N_{O}+I_{O}}}\right)
$$

For chirped-FSK, the receiver will only be affected by interference with probability $r_{W}$ and will not be affected with a probability of $\left(1-r_{W}\right)$. So the probability of error in the presence on the interference is:

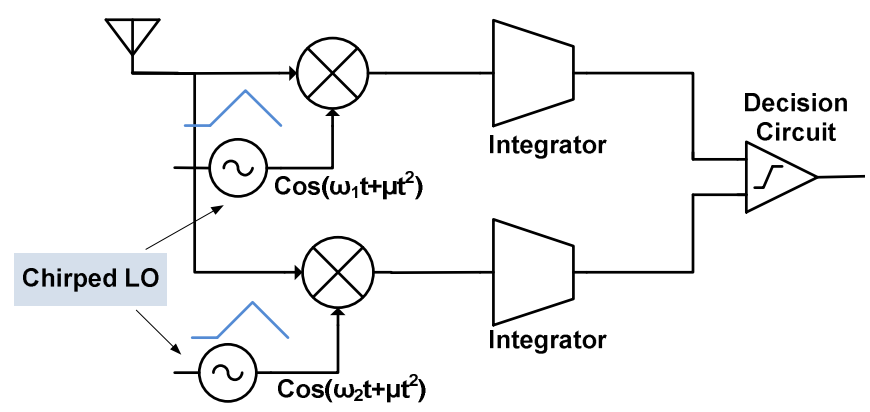

Figure 5 : chirped BFSK coherent receiver

$$
P_{C F S K-C H}=r_{W} Q\left(\sqrt{\frac{E_{b}}{N_{O}+I_{O}}}\right)+\left(1-r_{W}\right) Q\left(\sqrt{\frac{E_{b}}{N_{O}}}\right)
$$

If the interference bandwidth is $B_{i}$, then we can write,

$$
\frac{E_{b}}{I_{O}}=\frac{P_{S} T_{b}}{I_{O}}=\frac{P_{S} / R_{b}}{P_{I} / B_{i}}=S I R \frac{B_{i}}{R_{b}}
$$

where signal and interference power are $P_{S}$ and $P_{I}$ respectively and their ratio $(S I R)$ is the signal to interference ratio. Using this, (13) can also be written as:

$$
P_{C F S K-C H}=r_{W} Q\left(\sqrt{\frac{1}{S N R^{-1}+S I R^{-1} \frac{R_{b}}{B_{i}}}}\right)+\left(1-r_{W}\right) Q(\sqrt{S N R})
$$

\section{2) Non-coherent BFSK modulation}

Coherent FSK demodulation has the disadvantage that it needs time consuming phase synchronization along with frequency synchronization. Spending more time on synchronization means more energy consumption. One solution for this problem is non-coherent FSK detection. A non-coherent FSK receiver using two band-pass filters [13] is not a practical solution because of the very high-Q filter requirement. Another non-coherent FSK demodulator using a quadrature clock is shown in Figure 6 [12]. The probability of error of ideal non-coherent FSK detection is given by [13]:

$$
P_{N C F S K}=\frac{1}{2} \exp \left(-\frac{E_{b}}{2 N_{O}}\right)
$$

In the presence of interference and using a chirped clock for all the clock generators in Figure 6, the probability of error can be calculated as done for the coherent-FSK:

$$
P_{N C F S K-C H-I}=\frac{r_{W}}{2} \exp \left(\frac{-E_{b}}{2\left(N_{O}+I_{O}\right)}\right)+\frac{\left(1-r_{W}\right)}{2} \exp \left(\frac{-E_{b}}{2 I_{O}}\right)
$$

\section{B. Chirped-BPSK}

Binary phase shift keying (BPSK) is another popular modulation scheme with a good BER performance compared to BFSK. For our case, we use two chirped signals with opposite phase as the modulating signal: 


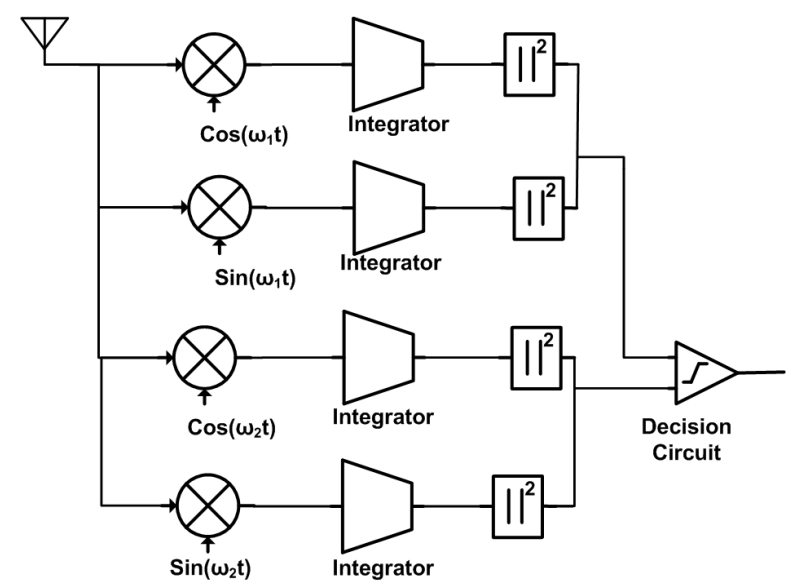

Figure 6 : Non-coherent BFSK detection by quadrature signals

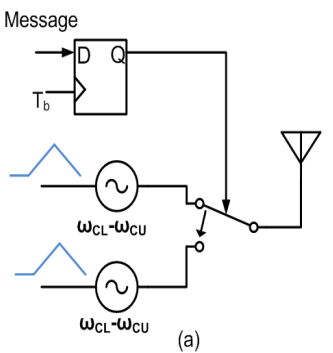

Figure 7 : BPSK modulator(a) and coherent detection(b) by single correlation

$$
s_{1}(t)=A \cos \left(2 \pi f_{0} t+\frac{1}{2} \mu t^{2}+a_{k} \pi\right)
$$

where $a_{K}$ represents the binary data. The BPSK modulation can be done by two clock generators which maintain a phase difference $\pi$.

The BPSK coherent detector, shown in Figure 7b, is simpler than the coherent BFSK demodulator. However, it also needs phase and frequency synchronization for a proper operation. We assume the proper phase and frequency synchronization is done. In that case the probability of bit error in the presence of white Gaussian noise can be written as:

$$
P_{P S K}=Q\left(\sqrt{\frac{2 E_{b}}{N_{O}}}\right)
$$

The effect of interference can be estimated as done in case of FSK modulation. The error probability when a chirped clock used in BPSK, can be written as:

$$
P_{P S K C H-I}=r_{W} Q\left(\sqrt{\frac{2 E_{b}}{N_{O}+I_{O}}}\right)+\left(1-r_{W}\right) Q\left(\sqrt{\frac{2 E_{b}}{N_{O}}}\right)
$$

\section{Simulation Results}

The bit error rate (BER) performance of the modeled system is simulated in MATLAB. Figure 8 shows the simulation block diagram where white gaussian noise and noise-like gaussian partial band interference is added to the transmitted signal in the channel. A random stream of bits with equal probability of ' 0 'and ' 1 ' is fed into the transmitter. The received message in

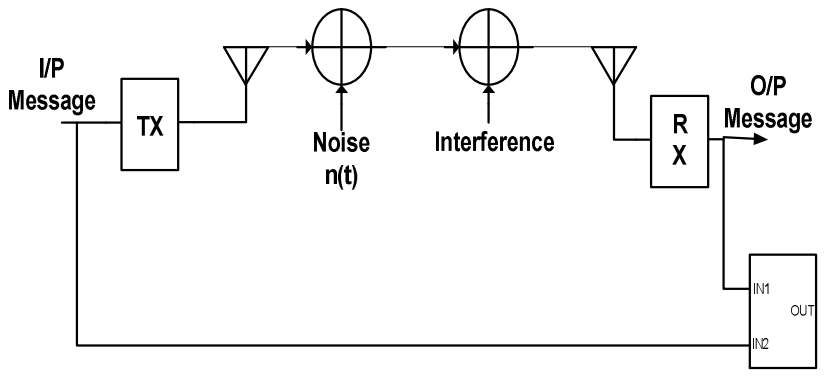

BER tool

Figure 8 : Model for system simulation

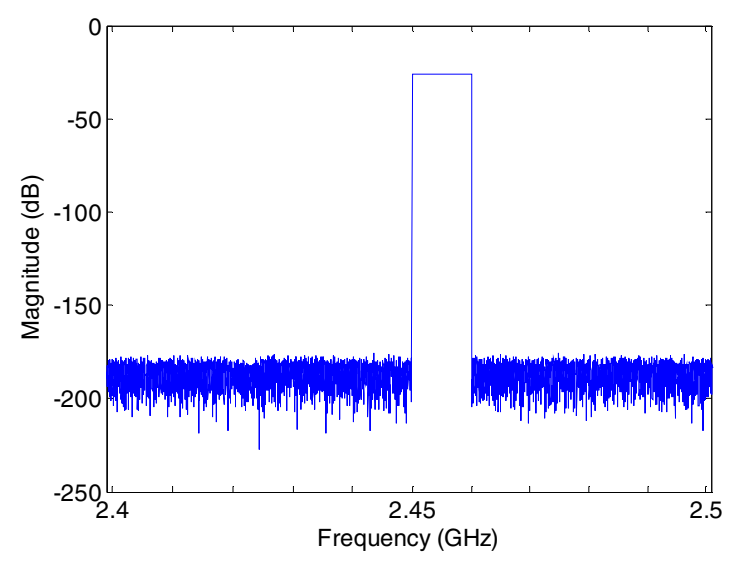

Figure 9 :Partial band Interference in frequency domain

the receiver from this corrupted signal is compared with the input message to calculate the BER as shown in Figure 8.

\section{A. Generating partial band noise like interference}

To simulate the interference robustness of the system an interference-model is created. Noise like Gaussian interference can be generated by a series of narrowband frequency tones starting from frequency $f_{i l b}$ where their phase, is a uniformly distributed random variable:

$$
S_{i}=\sum_{i=1}^{M} A_{i} \cos \left(2 \pi\left(f_{i_{-} l b}+\Delta f_{i} \frac{j}{M}\right) t+\phi\right)
$$

Where $\Delta f_{i}$ is interference bandwidth and $A_{i}$ is the amplitude of the signals generating interference. The number of frequency tones required depends on the sampling frequency and total number of time sample points used. Two consecutive sinusoidal tones should have integer number of cycles in the total number of points. If the sampling frequency if $f_{s}$ and total number of point in the time domain is $N$, then the frequency resolution is:

$$
f_{R}=\frac{f_{S}}{N}
$$

So, the number of frequency tones to be added for an interfering bandwidth of $\Delta f_{i}$ is:

$$
M=\frac{\Delta f_{i}}{f_{R}}=N \frac{\Delta f_{i}}{f_{S}}
$$

For an interference band of $10 \mathrm{Mhz}$, in the frequency range between $2.45 \mathrm{GHz}$ and $2.46 \mathrm{GHz}$, with sampling frequency of $10.24 \mathrm{GHz}$, the total number of points is, $N=2^{22}$ from (23), 


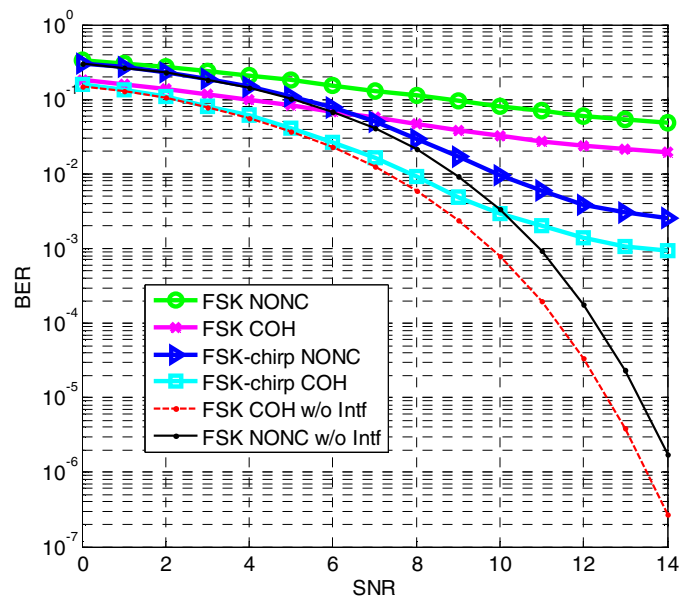

Figure 10 :Simulated BER of BFSK and chirped-BFSK in the presence of noise and interference (Low)

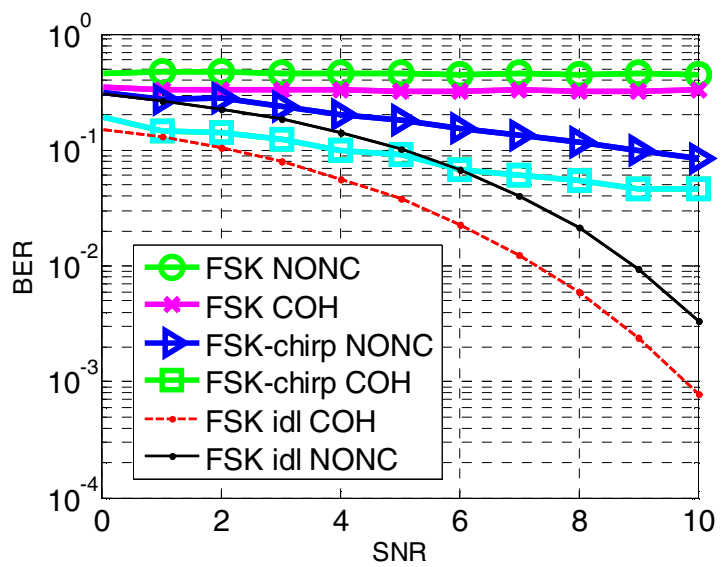

Figure 11 : Simulated BER of BFSK and chirped-BFSK in the presence of noise and interference (high)

number of frequency tones to be added, $M=4096$. The corresponding interference is shown in Figure 9.

The power of the interfering signal plays an important role in the system performance. The total power of the set of interfering signals given in (21) can be written as

$$
P_{i}=\sum_{n} x^{2}(t)
$$

The average power of $M$ sinusoidal signals with amplitude $\mathrm{A}_{\mathrm{i}}$ is:

$$
P_{A V G}=\frac{\left(A_{i}\right)^{2}}{2} M
$$

To achieve an interfering power of $P_{A V G}$ we need the signals to be:

$$
S_{i}=\sqrt{\frac{2 P_{A V G}}{M}} \sum_{i=1}^{M} \cos \left(2 \pi f_{i} t+\phi\right)
$$

\section{B. Chirped-FSK and chirped-PSK results}

The FSK coherent and non-coherent systems are simulated in MATLAB for their BER performance. The clocks are assumed synchronized accurately between the RX and the TX. The performance is similar to the ideal coherent and noncoherent receivers respectively. We choose a frequency difference and bit rate both $5 \mathrm{MHz}$. An interference band of

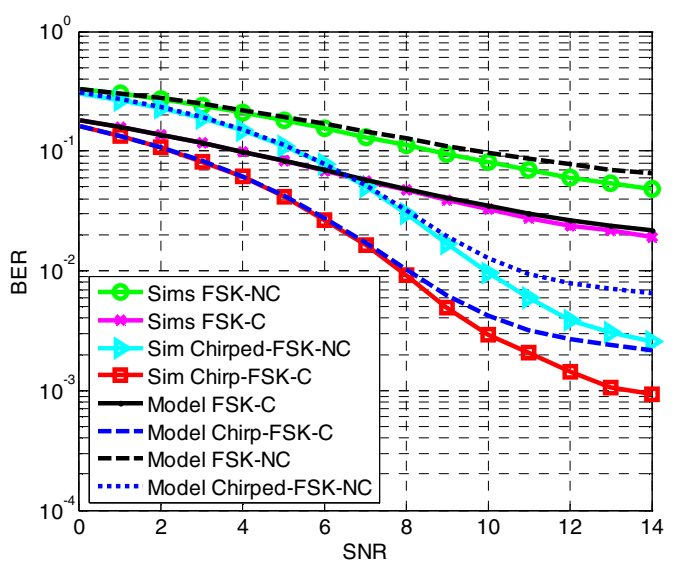

Figure 12 : Simulated and Modeled BER comparison for BFSK and chirped-BFSK in the presence of noise and interference

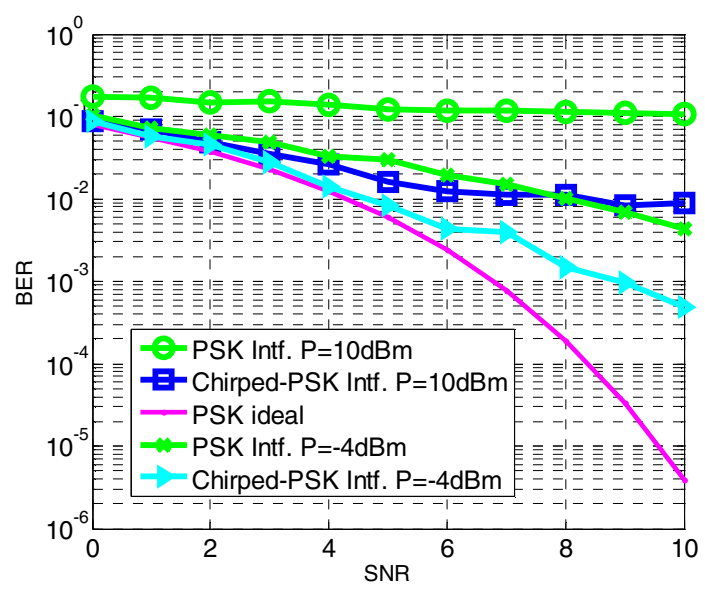

Figure 13 : Simulated BER of BPSK and chirped-BPSK in the presence of noise and interference

$10 \mathrm{MHz}$ from $2.45 \mathrm{GHz}$ to $2.46 \mathrm{~Hz}$ is added in the channel. We simulate for two interference power levels of $-4 \mathrm{dBm}$ (Intf-1) and $10 \mathrm{dBm}$ (Intf-2) when signal power in $0 \mathrm{dBm}$. The two FSK carriers for ' 0 'and ' 1 ') are at $2.452 \mathrm{GHz}$ and $2.457 \mathrm{GHz}$. Comparison of performance for both coherent and noncoherent detection are shown in Figure 10 and Figure 11 for intf-1 and intf-2 respectively. The conventional FSK performance is more affected by interference compared to the chirped FSK performance as can be observed. The simulated performance is compared with equations (13) and (17) and are shown in Figure 12. Up to approximately $8 \mathrm{~dB}$ SNR, the simulated values match well with the theoretical values. For higher SNR values the results differ. This is still under investigation. The BPSK system shown in Figure 7 is simulated in MATLAB. with similar chirp characteristics. One single frequency of $2.45 \mathrm{GHz}$ is used for BPSK modulated signal. When a perfectly synchronized clock between the TX and the RX is used, the BER of both the systems overlap (not shown in the figures). This means that the same PSK system can be used for chirped-BFSK given a proper chirp generator and synchronization scheme is in place. Interferences with similar characteristics (as used for FSK) are added in the channel. The simulated BER performance is plotted in Figure 13. In the presence of interference, the BER is more severely 


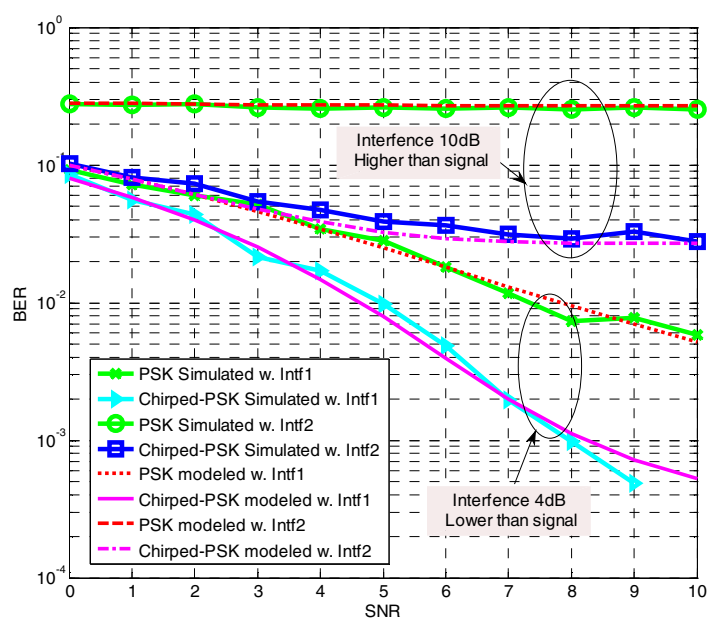

Figure 14 : BPSK and chirped-BPSK simulated and modeled BER comparison in the presence of noise and interference

degraded for BPSK as compared to the chirped-BPSK. The predicted probability of error is indeed a close approximation as can be seen from the Figure 14. These simulation results illustrate that by using a chirped carrier the BFSK and BPSK systems can be made interference robust.

\section{CONCLUSION}

A Chirped frequency clock is used to spread the spectrum and thereby achieve interference robustness. We show by simulation that the correlation based ideal BFSK and BPSK detectors perform similar for a chirped communication system when a synchronized chirped clock is used to make it chirpedFSK and chirped-PSK respectively in the presence of white gaussian noise. The BER of the BFSK and chirped BFSK are compared in the presence of noise and partial band interference. Chirped-BFSK shows better BER performance than non-chirped BFSK, as predicted by the BER model. We did similar simulations for BPSK transceiver system. This proves that a chirped clock can provide interference robustness to BFSK and BPSK systems. To get interference robustness at a low power consumption, a comparison of FSK demodulation architectures has to be done to tradeoff power consumption and BER performance.

\section{REFERENCES}

[1]R. Dutta, R. v. d. Zee, M. J. Bentum and A. B. J. Kokkeler, "Choosing Optimum Noise Figure and Data Rate in Wireless Sensor Network Radio Transceivers," International Conference on Communications (ICC), 2011.

[2]I. F. Akyildiz, et al., "A survey on sensor networks," Communications Magazine, IEEE, vol. 40, pp. 102-114, 2002.

[3]M. Petrova, et al., "Performance study of IEEE 802.15.4 using measurements and simulations," in Wireless Communications and Networking Conference, 2006. WCNC 2006. IEEE, 2006, pp. 487-492.

[4]J. Oetting, "A Comparison of Modulation Techniques for Digital Radio," Communications, IEEE Transactions on, vol. 27, pp. 1752-1762, 1979.

[5]M. B. Pursley, "Role of spread spectrum in packet radio networks ," Proceedings of the IEEE, vol. 75, pp. 116-134, 1987.

[6]J. G. Andrews, et al., "Ad Hoc Networks: To Spread or Not to Spread? [Ad Hoc and Sensor Networks]," Communications Magazine, IEEE, vol. 45, pp. 84-91, 2007.

[7]J. Q. Pinkney, et al., "A robust high speed indoor wireless communications system using chirp spread spectrum," in Electrical and Computer Engineering, 1999 IEEE Canadian Conference on, 1999, pp. 84-89 vol.1.
[8]C. E. Cook and M. Bernfeld, Radar signals: an introduction to theory and application: Artech House, 1993.

[9]M. R. Winkler, "Chirp Signals for Communcation," Wescon Convertion Record, vol. 7, 1962.

[10] M. Roberton and E. R. Brown, "Integrated radar and communications based on chirped spread-spectrum techniques," in Microwave Symposium Digest, 2003 IEEE MTT-S International, 2003, pp. 611-614 vol.1.

[11] M. A. Richards, James A. Scheer, William A. Holm, "Principles of modern radar," Raleigh, NC : SciTech Pub, 2010.

[12] F. Xiong, "Digital Modulation Techniques," Artech House, vol. 2nd ed. Boston/London, 2006.

[13] J. G. Proakis, "Digital Communication," McGraw-Hill, 2000. 